\title{
Ventricular Fibrillation Caused by Hypokalaemia During a Diabetic
}

\section{Ketoacidosis}

\author{
Newman $C^{1 *}$, Costello $M^{1}$, Quinn $S^{2}$, Bates $\mathbf{J}^{3}$, McNicholas $B^{3}$, McNicholl $B^{4}$, O'Keeffe $D^{1}$, O'Shea $P^{5}$ and \\ O’Sullivan $\mathbf{E}^{1}$
}

${ }^{1}$ Department of Endocrinology, Diabetes and Metabolism, Galway University Hospital, Newcastle, Galway City, Ireland ${ }^{2}$ Department of Cardiology, Galway University Hospital, Newcastle, Galway City, Ireland

${ }^{3}$ Departments of Anaesthesiology and Critical Care, Galway University Hospital, Newcastle, Galway City, Ireland

${ }^{4}$ Department of Emergency Medicine, Galway University Hospital, Newcastle, Galway City, Ireland

${ }^{5}$ Department of Clinical Biochemistry, Galway University Hospital, Newcastle, Galway City, Ireland

"Corresponding author: Newman C, Department of Endocrinology, Diabetes and Metabolism, Galway University Hospital, Newcastle, Galway City, Ireland, Tel: +353 91524 222; E-mail: christinenewman89@ gmail.com

Received: September 28, 2019; Accepted: October 07, 2019; Published: October 17, 2019

\begin{abstract}
Background: Diabetic ketoacidosis is a common and potentially life-threatening endocrine emergency. Due to broad recognition and protocolised treatment regimes, the mortality rate remains at $<1 \%$. Markers of serious disease include hypokalaemia, severe acidosis, reduced Glasgow Coma Scale and uraemia.

Patient: We describe the case of a 40-year-old gentleman presenting with acidosis, ketosis, hyperglycaemia and a potassium level below the recordable reference range of our assay. Due to a severe acidosis, an acute kidney injury, severe hypotension and anuria, treatment with the DKA protocol was initiated. In addition to fluid resuscitation the patient received a stat dose of 6 units intravenous insulin and was commenced on a weight based infusion. Multiple arterial blood gas samples did not provide a numerical value for the potassium level, and the patient was treated with IV fluids for over 2 hours before a serum sample confirmed profound hypokalaemia. The patient developed ventricular fibrillation shortly after and died despite advanced cardiac life support, intubation, magnesium replacement and aggressive potassium replacement.

Discussion: Hypokalaemia is an uncommon presentation in DKA and is present in only 5\% of cases presenting to Emergency Departments (ED). Despite this, its presence is a poor prognostic indicator as the hypokalaemia is worsened by the administration of insulin. It is essential that potassium levels be ascertained prior to the administration of insulin.
\end{abstract}

Keywords: Diabetic ketoacidosis; Cardiac arrest; Hypokalaemia

Citation: Newman C, Costello M, Quinn S, et al. Ventricular Fibrillation Caused by Hypokalaemia During a Diabetic Ketoacidosis. Clin Case Rep Open Access. 2019;2(4):136.

(C)2019 Yumed Text. 
www.yumedtext.com | October-2019

\section{Learning Points}

- Clinicians should be aware that hypokalaemia is present in 5\% of DKAs and is a poor prognostic indicator.

- Its presence indicates significant osmotic diuresis and secondary hyperaldosteronism.

- Potassium levels should be confirmed prior to the commencement of insulin which serves to drive potassium intracellularly and exacerbate the effects of hypokalaemia.

\section{Case Description}

A 40-year male was brought by ambulance to the emergency department following a loss of consciousness. On arrival his capillary blood glucose was $>30 \mathrm{mmol} / \mathrm{L}$ and ketones were elevated at $3.4 \mathrm{mmol} / \mathrm{L}$. An ABG confirmed a blood glucose level of $60 \mathrm{mmol} / \mathrm{L}$, $\mathrm{pH}$ of 6.918 , bicarbonate of $3.2 \mathrm{mmol} / \mathrm{L}$ and lactate of $0.8 \mathrm{mmol} / \mathrm{L}$. There was no numerical value available for potassium on point-of-care testing.

\section{Methods and Procedures}

Treatment included 2 litres of normal saline and a stat dose of IV insulin followed by an infusion of $0.1 \mathrm{unit} / \mathrm{kg} / \mathrm{h}$. Serial ABGs were performed however a numerical value did not appear beside the potassium reading and it was assumed the patient was hyperkalaemic with a potassium value above the upper limit of the reference range.

Normal saline without potassium was continued and he received a stat dose of $10 \mathrm{ml}$ of $10 \%$ calcium gluconate (despite a reassuring ECG - FIG. 1a). Approximately 2 hrs after presentation, central laboratory serum values were available to the treating team. Serum potassium concentration was reported as below the lower limit of the reference range $(<1.5 \mathrm{mmol} / \mathrm{L})$ and urea and creatinine levels were raised at $18.9 \mathrm{mmol} / \mathrm{L}$ and $273 \mu \mathrm{mol} / \mathrm{L}$ respectively. Potassium replacement was initiated but the patient's clinical condition rapidly altered (as evidenced by a repeat ECG -FIG. 1b showing global ST segment depression). The patient developed ventricular fibrillation and died despite Advanced Cardiac Life Support and aggressive potassium replacement of $320 \mathrm{mEq}$.

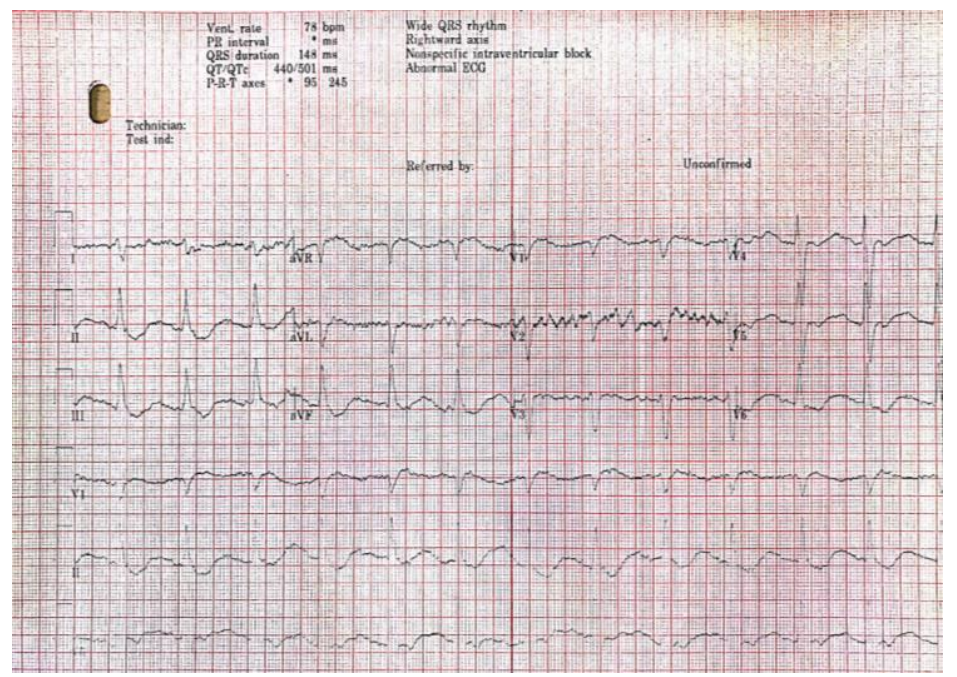

FIG. 1a. Represents a reassuring ECG taken at the time of arrival in ED. 


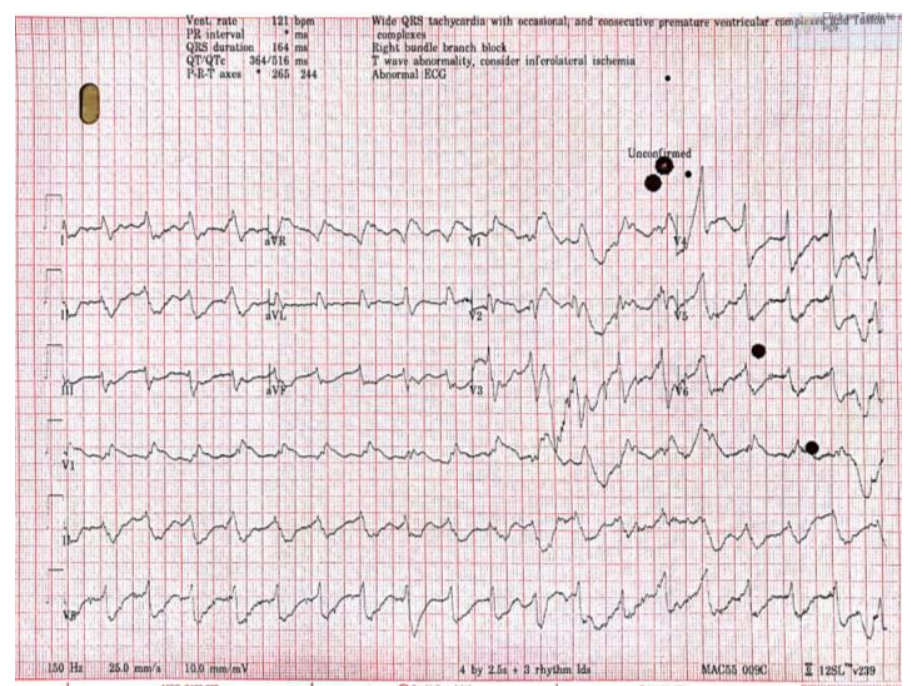

FIG. 1b. Represents a significant change with tachycardia, a wide QRS complexes and wide-spread ST depression.

\section{Discussion}

The majority of patients presenting with DKA have a potassium deficiency of 3-5 $\mathrm{mEq} / \mathrm{kg}$ due to renal losses and vomiting [1]. Despite this, the deficit is often not reflected in serum samples as potassium moves from the intracellular space extracellularly in the presence of acidosis. This can conceal the true extent of potassium depletion and hypokalaemia is seen in only $5 \%$ of DKAs at presentation [2]. Point-of care testing may also be misleading as discrepancies ranging from -0.02$0.06 \mathrm{mmol} / \mathrm{L}$ for potassium have been documented between $\mathrm{ABG}$ and central laboratory levels [3].

Hypokalaemia is recognised as a marker of severe DKA and the majority of treatment guidelines recommend replacing potassium to serum concentrations of at least $3.3 \mathrm{mmol} / \mathrm{L}$ prior to initiating insulin treatment. This is because insulin activates sodium-potassium ATPase channels causing a shift of potassium into cells [4] worsening any preceding hypokalaemia and complications may include fatal arrhythmias.

A retrospective review of this patient's notes revealed that unbeknownst to the treating team, he had sought investigation for longstanding diarrhoea in the months prior to his presentation. This potentially contributed to the profound hypokalaemia. Outside of hypokalaemia this patient displayed many other indicators of poor prognosis including first presentation and uraemia [5].

In summary, despite protocolised treatment and a mortality rate of 1\%-2.4\%, DKA can be fatal, and levels of potassium should be ascertained prior to instituting insulin therapy. It is also strong advisable to use central laboratory levels where possible to eliminate any discrepancies between point of care and central levels.

\section{Conclusion}

In summary, DKA is a common presentation in emergency departments. Though the frequent use of approved protocols has reduced the mortality rate, complex and diverse cases can still present which require senior and multi-disciplinary input. Hypokalaemia is once such instance and as demonstrated in this case can prove fatal. 
www.yumedtext.com | October-2019

\section{Conflicts of Interests}

None of the authors have any conflicts of interest in relation to this case report.

\section{Acknowledgements}

None.

\section{REFERENCES}

1. Liamis G, Liberopoulous E, Barkas F, et al. Diabetes mellitus and electrolyte disorders. World J Clin Cases. 2014;2(10):488-96.

2. Arora S, Cheng D, Wyler B, et al. Prevalence of hypokalaemia in ED patients with diabetic ketoacidosis. Am J Emerg Med. 2012;30(3):481-4.

3. Leino A, Kurvinen K. Interchangeability of blood gas, electrolyte and metabolite results measured with point-ofcare, blood case and core laboratory analyzers. Clin Chem Lab Med. 2011;49(7):1187-91.

4. Li T, Vijayan A. Insulin for the treatment of hyperkalaemia: a double-edged sword? Clin Kidney J. 2014;7(3):23941.

5. Venkatesh B, Pilcher D, Prine J, et al. Incidence and outcome of adults with DKA admitted to ICUs in Australia and New Zealand. Crit Care. 2015;19:451. 